\section{CLINICAL LECTURE ON} SOME URINES AND URINARY CALCULI.

\author{
By WILLIAM M. ORD, M.B.LOND., F.R.C.P., \\ Senior Assistant-Physician, St. Thomas's Hospital.
}

Gentlemen,-I propose to-day to review some of the more interesting urinary specimens that have come before us of late, associating with them such illustrative matter brought from without as we have freely used in our joint efforts to make the observations of the out-patient room more useful.

1. Condition of the Urine in a Case of Poisoning by Iodine.- "Poisoning" is perhaps rather a strong word, if the effects usually following the swallowing of the tincture of iodine be considered. As far as I know-and I have seen here and elsewhere several cases in which the tincture has been taken by mistake or accident-the most serious result is a sharp irritation of the mucous membrane in the path of the fluid. Vomiting is induced when the fluid reaches the stomach, and, though salutary in the most important respect, involves, of course, further contact of the regurgitate iodine with the tender faucial membranes.

F. Y., a boy aged 3, was admitted into Alice Ward on August 28th, 1876. All the history that could be obtained was to the effect that he had swallowed some tincture of iodine from a bottle in his mother's room, and that he had vomited immediately afterwards. The quan. tity swallowed and the proportion rejected were unknown. Dr. Sharkey (resident assistant-physician) found the child to be suffering with croupy cough, and admitted him by way of precaution. Otherwise than in experiencing great irritation of the mouth and throat, the child did not appear to have taken any harm. The croupy cough passed away as the throat healed, and the child was discharged well at the end of nine days.

Having seen recently a note in the BRITISH MrdicAl JOURNAI relating that Dr. Simon had found albumen in the urine of a patient after the application of iodine to the skin, I requested my clinical clerk, Mr. Gulliver, to examine some urine passed by the child the day afte admission; that is to say, the day after the accident. Mr. Stewart of the chemical laboratory, was good enough to assist Mr. Gulliver. They found the urine amber-coloured, of specific gravity 1038, somewhat strongly acid, throwing down on cooling a slightly flocculent sediment, amorphous under the microscope.

The addition of acetic or nitric acid to the urine produced a copious amorphous precipitate, soluble in excess of nitric acid and on the application of gentle heat. On subsequent cooling, a precipitate again fell, and was found to consist of yellow halberts of uric acid mixed with fine granular matter. No precipitate was obtained on boiling; and Mr. Stewart, who at first suspected the presence of some modified form of albumen, states that, on further examination, he detected nothing of the kind. There was large excess of urea. Mr. Stewart obtained good reactions of iodine, combined, not free, and of indican. I may remark in passing that the test used for iodine was a double test. In one, starch mucilage was used first, and nitric acid added afterwards. Good indications were also obtained with the process I now exhibit. A few drops of bisulphide of carbon are placed in a test-tube, which is then half filled with urine. If, on shaking, the bisulphide become tinted with a rose colour, iodine is present free. If, again, the tint appear after the addition of chlorine water or strong nitric acid, iodine is present in combination. The tint here does not appear till after the addition of nitric acid, and iodine is, therefore, present in combination.

Now, when uric acid separates from urine in the halbert form, there is always reason to suspect the presence of some considerable quantity of colloid matter. I have shown this in former papers, and have demonstrated it again and again in the out-patient room. In order to push further the question of the presence of colloids, I obtained some urine passed a day later. The specific gravity had fallen to 1025 ; the other reactions were as before. Failing to obtain indications of albumen by the ordinary means, I added to a small bulk of the urine (the whole quantity at my disposal was under two ounces) an equal bulk of absolute alcohol. Two samples of average urine (one of specific gravity 1019, one of 1026) were treated in the same way at the same time. The cloud produced at once in the boy's urine was much denser than that in the others, and gathered at the end of a week to a flocculent mass occupying nearly a fifth of the fluid, the precipitate in the others being a little powder hardly visible at the bottom of the test-tubes. This precipitate gave the reactions of mucin, and not of albumen. No tint was produced on heating with Millon's reagent. Under the microscope, it consisted of brilliant spherules with long colourless prisms, a mixture of urates and phosphates in a finely granular adherent substance, mucin. The colloid giving the distinctive shape to the crystals must in this case have been mucus present in unusually large quantity dissolved or in cumplete hydration.

$\AA$ word on the nitric acid reaction in this case. The test in which a stratum of cold nitric acid is brought into contact with a superjacent stratum of urine is of much use in the detection of the presence of small quantities of albumen, and has, as you are aware, been put to the purpose of quantitative determination of albumen in urine by that excellent practical observer Dr. W. Roberts of Manchester. It occasionally happens, as in this case, that uric acid is thrown down even at the ordinary temperature of rooms and laboratories, and this circumstance makes it desirable in all cases to correct the experiment by further observation : a matter of no difficulty. But I may further point out to you that, after a little practice, you will be able to distinguish between albumen and uric acid by the formation of the cloud. In the case of the albumen, the cloud is horizontal and of uniform thickness. Usually one or two lighter clouds are formed above with clear fluid between. These are perfectly parallel and uniform strata. With uric acid the cloud is irregular on its upper surface, like a storm-cloud. It extends with some rapidity to the rest of the fluid, like the same storm-cloud mounting the sky, and never, as far as I know, do parallel strata appear above.

2. Case of Spontaneous Disruption of Calculi.-The specimens exhibited were passed by a gentleman aged 84 , who, three years ago, weighed $25 \mathrm{st}$. He had been in his early days a hard worker and, as he alleged, a small eater, particularly of meats. For the last twenty years, however, he had been leading a sedentary life, chiefly because of his bulk. His general health has been good, and, though there is gouty enlargement of his knuckles, he has never had any acute gouty or rheumatic illness. One day, when passing urine, he observed two round stones fall into the chamber-utensil he was using. He was rather amused than frightened, and, feeling no inconvenience, took no notice of the matter. Not long afterwards, his urethra was for some hours obstructed by a calculus. A catheter was introduced, but only as far as the calculus, which barred the way to the bladder. The stone ultimately came away, and was, like the first passed, perfectly spherical. For a year after this, his health was bad. He lost all appetite, took very little food, and wasted considerably. Then he begar to pass stones in fragments. The number passed was very large, enough, as he said, to gravel the path to his house. $\mathrm{He}$ is not aware that there was any change in the quality of his urine. He felt no unusual sensations in the bladder; nothing like an explosion. He had no violent fall or jerk that he remembers. Most of the stones thus passed were in fragments.

After the stones had come away, his health underwent a fresh change. He was attacked with eczema and senile prurigo, and at the same time regained his appetite./ Then he began to grow fat again, and his legs, which had become œdematous, resumed their natural state.

At present, he is well and cheerful, his only complaint being of " heaps of sand" in his eyelids. His urine, otherwise healthy, always contains crystals of uric acid, and sometimes small friable concretions of the same. The calculi exhibited are only a few out of hundreds that he has passed ; some are round, some irregular; but it is evident that the majority are fragments of larger calculi ; they are such fragments as might be compared to fragments of an exploded spherical shell. Each has a pyramidal form, with the base of the pyramid convex, the apex slightly hollowed. The calculi of which they would appear to have formed parts must have been mostly about half to threequarters of an inch in diameter, and each sphere must have split up from the centre outwards into seven or eight segments. It is evident, on close examination, that the fracture occurred some time before the stones were voided. The surfaces are not fresh or sharp, and the whole stone is covered with an uniform grey deposit. A section being carried in a radial direction through any one of them, the surface exposed is marked by concentric lines, by fragments of a concentric system of which the rounded base would be the outermost circle, and of which the centre would be the apex of the fragment, if it remained perfect. Such a section demonstrates at once the fact that the seeming segments are, in fact, segments of a calculus built up of many concentric layers. The section-surface is of a reddish grey colour, very compact, and exhibits no cracks or irregularities of consistence. The crust which invests the whole fragment, and which is moulded 
to all the irregularities left by the fracture, is much lighter in colour than the substance of the calculi. (See Fig. I.)

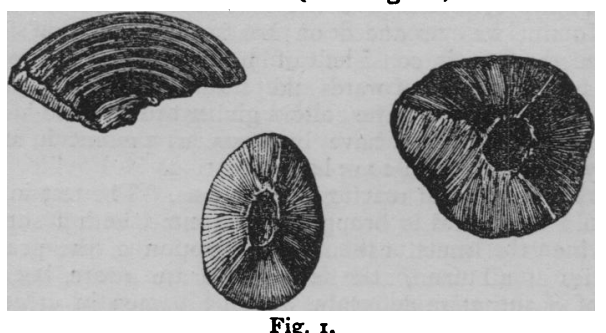

Fig. x.

The calculi are composed of uric acid and urate of ammonia intimately mixed. The outer light portion, which has the look of a phos. phatic deposit, is, in fact, urate of ammonia. When this is dissolved in liquor potassæ, it leaves a gelatinous matrix and a great abundance of spores and mycelium, strongly resembling those of microsporon furfur. The central portion of the original calculus is wanting in all the fragments. Under the microscope, the mass of the calculus is found to consist of compact crystalline matter with a radial fibration. No separate crystals are to be obtained.

The cause of the original fracture of the calculi appears to me to have been an expansion of the central portion, or some expansion at the centre, acting as the exploding powder in a shell. There is evidence that, for some time preceding the appearance of the fragments, there was enough disturbance'of the general health to render it probable that the chemical constitution of the urine was altered. There is also evidence in the nature of the secondary deposit that the state of the urine was different after the fracture from the state accompanying the formation of the calculi. I infer that such change was accompanied with a tendency to alkalinity, and that as a result a chemical influence was exerted upon the calculi, tending to cause swelling of any mucoid matters which might enter into their composition; that the centre which has disappeared was different in some respects from the circum. ference which remains; and that this difference determined an unequal swelling of the centre, resulting in disruption of the spheres.

That this was the case rather than a disintegration or falling to pieces through general failure of cohesion, is an idea supported by the perfection of the individual segments and the compactness of their structure. The regular form of the fragments opposes the idea of fracture having arisen through collisions among calculi. Such conditions would produce irregular chipping rather than radial fissure and segmentation. An inspection of the fragments resulting from lithotripsy will show the essential nature of the difference. It is not uncommon, indeed, to find calculi that have been cut in two and preserved, as eommonly found in museums, fissured radially round the centre. This is, to my mind, usually an acquired, and not an originally present, condition, not existing when the calculi were in the bladder, due to the unequal drying of the centre and circumference. The condition, however, indicates that under exposure to certain circumstances of altered moisture, radial splitting, beginning from the centre, may occur in calculi ; and what I suggest is, that a similar sort of process led to the segmentation in this case under circumstances which determined swelling instead of shrinking of the nucleus.

The spontaneous fracture of vesical calculi is apparently not a common event. The late Mr. Southam, however, published in the BRITISH MEDICAL JouRnal two cases observed by himself and one of Mr. Luke, quoting also a case of $\mathrm{Mr}$. Liston and giving a drawing of a calculus from the Dupuytren Museum of Paris. The paper was illustrated by excellent drawings, which have been reproduced in Dr. Beale's work on the Urine. The calculi in Mr. Southam's two cases were larger than those now exhibited, and the hypothesis of rupture by internally developed gas was advanced. The fragments were only two in each case, and were less regular segments of spheres. One was composed of lithic acid with a coating of hard oxalate of lime, and the fragments, after disruption, had become coated with fresh phosphate. It is, therefore, quite possible that changes in the composition of the urine played a part in producing disruption. The composition of Mr. Southam's second calculus is not stated. In Mr. Luke's case, the calculus was composed of triple phosphate, and there was no history of violence. In Mr. Liston's case, the calculus is supposed to have broken up suddenly under the influence of a violent bodily shock. The fragments were numerous, and the result was fatal. No analysis is given. In the last case only the drawing is given, showing a fragment separated by fissures, but not detached. The fissures do not reach the nucleus, and the fragment is held in place by fresh enveloping deposit.
Mr. Coulson has also recorded a case in which a sort of natural lithotripsy occurred, two or more calculi being crushed together and broken by one another as nuts may be broken in the hand. In such cases, the calculi are mostly of loose consistence, and the fragments are irregular and not segmented. (Pathological Transactions, vol. xv, p. 143.)

I have been fortunate enough to find in the Museum of the Royal College of Surgeons a preparation (Calculi, A 177) very closely resembling my own. It is figured at the end of the catalogue in Plate I, figs. 6,7 , and 8 . There is no history, but the preparation is described as a pisiform calculus (Prout's term) "separated into triangular portions, possibly the result of spontaneous disintegration". The seg. ments are pointed, instead of being, like these, truncate ; that is to say, the centre remains. They are composed, according to Mr. Taylor's analysis, of uric acid, and appear to have been coated by fresh material after fracture.

In relation to the analysis of these calculi, I may add a few remarks illustrating the use of the microscope in these processes. After the general facts of organic composition, of the presence of uric acid and ammonia had been determined, some of the central part was chipped and examined under the microscope. The characters of the fragments have been already noted, and it may be observed that the whole of the original portion of the calculus was built up of fine concentric laminæ, perfectly compact, perfectly continuous with one another, and showing a radial crystalline structure. The superficial portion, on the other hand, consisted of spherules imbedded in a clear matrix, the spherules being dense at their centre, less dense and lighter in colour, with marked radial crystalline structure, at their circumference. This is the form taken by urate of ammonia in the presence of colloids. The spheres were slowly disintegrated by hydrochloric acid, and rapidly dissolved by caustic potash, leaving the matrix and the contained fungoid structures.

The central mass, on being powdered and boiled in distilled water, dissolves sparingly, giving a strongly acid solution, from which large very thin flat lancet-ended crystals are thrown down. These are exactly like the crystals which $I$ have shown elsewhere to be produced when uric acid is added in large excess to hot solutions of urate of ammonia. As I believe them to be crystals of a highly acid urate, they will indicate that only a comparatively small relative quantity of ammonia is present. After three or four exhaustions of the powder with boiling distilled water, rectangular plates of pure uric acid are deposited. There is clearly, then, also a large proportion of uncombined uric acid, representing, in fact, more than half the bulk of the calculus. The solution yielded by the surface is neutral. The quantity of material being small, and the material itself comparatively soluble, crystallisation was not effected. No oxalate crystals were obtained in any process. [To be concluded.]

\section{A N A D D RES S ON \\ OPHTHALMOLOGY IN ITS RELATION TO GENERAL MEDICINE.}

The Annual Oration delivered before the Medical Society of London.

By J. HUGHLINGS JACKSON, M.D., F.R.C.P.,

Physician to the London Hospital, and to the Hospital for the Epileptic and Paralysed; etc.

III.

THERE are still other valuable deductions to be made from the facts of cases of ocular palsy. In a case of paresis of the right external rectus, when the patient tries hard to turn the lamed eye out, the other eye turns in too much; there is secondary deviation. From the attempt to move the paralysed or weak part, there is a double effect; there is, firstly, failure to move it, and, secondly, overmovement of associated muscles.

Later on, we shall see that there is evidence, both by cases of disease of the brain and by Ferrier's experiments, that there are centres for conjoint movements of both eyes. This principle bears in a most important way on the interpretation of locomotor ataxy and of disorders of co-ordination in general. It has already been applied by Wundt to explain the disorder of co-ordination produced by experiments on animals; but, so far as I know, it has not been yet applied by physicians to explain cases of disorder of co-ordination by disease. I do not, however, maintain that in locomotor ataxy there is solely a motor difficulty ; there is sensory defect, and the joints and bones even become diseased. Let us, however, apply the principle so far as it will 